\title{
HACIA UNA NUEVA HISTORIA DEL PUERTO DE ROSARIO \\ Y EL DESARROLLO REGIONAL. \\ RENOVACIÓN HISTORIOGRÁFICA Y \\ APORTE INTERDISCIPLINAR
}

\author{
Miguel Ángel De Marco (h)*
}

\begin{abstract}
Resumen
El presente aporte se refiere a las implicancias de los abordajes del puerto de Rosario desde la perspectiva ofrecida por la categoría de las "ciudades puertos regionales" en la integración de distintos campos historiográficos, enfoques interdisciplinarios y experiencias recientes en la ejecución de proyectos relacionados a la puesta en valor del patrimonio identitario de los puertos.
\end{abstract}

Palabras clave: historia, Rosario, región, puertos, interdisciplinariedad.

\begin{abstract}
The present contribution refers to the implications of the approaches of the port of Rosario from the perspective offered by the category of "regional ports cities" in the integration of different historiographical fields, interdisciplinary approaches and recent experiences in the execution of projects related to the Enhancement of the identity heritage of the ports.
\end{abstract}

Keywords: history, Rosario, region, ports, interdisciplinary

Cuando en 1962 se encontró en la desembocadura de Arroyo Seco restos de cerámica de varios siglos de antigüedad, el diario "La Capital" de Rosario, en una nota sin firma en su suplemento cultural, databa el origen de la arqueología santafesina en 1864, es decir, hace más de 150 años, cuando François Séguin, a metros de la desembocadura del río Carcarañá halló piezas y restos humanos de una antigüedad superior a los 4 mil años, según se relataba. La exposición a la que me voy a referir reconoce como punto nodal a esa etapa de la historia argentina, que es contemporánea a la llegada de los exploradores de fósiles como Bravard, Séguin, Bonnement, para luego venderlos o remitirlos a los museos europeos. En 1962, semanas después del hallazgo de Arroyo Seco, aquí mismo en Rosario, en un sector cercano al puerto, donde se estaba removiendo la tierra para construir lo que sería luego el Anfiteatro Humberto de Nito, se encontraron fósiles. Alertadas las autoridades se buscó a un especialista y se envió al señor Federico Henning, alemán residente en Santa Fe desde la década del 30 y que desde ese entonces se desempeñaba como coleccionista

\footnotetext{
* Investigador del Consejo Nacional de Investigaciones Científicas y Técnicas de la República Argentina (CONICET), Unidad IDEHESI-Nodo IH.
} 
del Instituto de Fisiografía y Geología de la Facultad de Matemáticas de la UNL. Para entonces, hacía ya veinte años que Agustín Zapata Gollán, en la misma Universidad trabajaba sobre Cayastá.

Estos hechos dispersos, los menciono intencionalmente a manera de ejemplo, para generar interrogantes que acerquen las inquietudes de su disciplina con la propuesta que plantearé en relación con analizar con método el círculo o la dinámica de las ciudades puertos regionales, y la necesidad de profundizar los estudios interdisciplinarios porque las distintas historias se explican mutuamente.

En primer lugar, quiero expresar mi agradecimiento a los organizadores de esta reunión académica por invitarme a compartir con ustedes un tema de mi especialización que requiere del abordaje de distintas disciplinas.

Estudiar las Ciudades Portuarias Regionales como Rosario, y aún el puerto en sí requiere en primer lugar destacar el rol de estas como "poleas" de las políticas económicas y las gestiones de gobierno en distintos Estados nacionales del siglo XIX y XX. En un segundo lugar subrayar las posibilidades que se desprenden de los estudios comparados e interdisciplinarios sobre la evolución de la ciudades portuarias.

Desde la historia económica se puede acceder a singulares perspectivas de la recepción y adhesión a proyectos de nación, disímiles y hasta hostiles entre sí.

La historia de las crisis en la Argentina ofrecen instancias para detectar el conflicto entre las tendencias proteccionistas y librecambistas y su repercusión en la política portuaria. Pero el estudio de estas tendencias a escalas más amplias permiten también analizar la repercución de las mismas en lo que refiere a la relación de los puertos con sus foreland y sus hinterland.

El especialista mexicano Carlos Martner Peyrelongue afirma que el proteccionismo y el librecambismo afecta la relación del puerto con el mundo y especialmente con su región. En tal sentido sostiene que la zona de influencia de los puertos en etapas proteccionistas redujeron su actividad y debilitaron sus contactos, y que el modelo de desarrollo latinoamericano basado en la industrialización por sustitución de importaciones había debilitado su posición, como punto de contacto, entre lo interno y lo externo, como lugar de intercambio mercantil y cultural. Mientras que los puertos con políticas de librecambio priorizando su relación e influencia externa había debilitado simultáneamente su zona de influencia interna, transformándose en una terminal de productos, almacenamiento y bodega.

En uno y otros sentidos, determinar la intensidad de los flujos y por ende de los contactos se relaciona en gran parte con estos lineamientos de comercio exterior.

Es mi caso personal, a través de proyectos y equipos en conformación, estoy especialmente interesarado en detectar la evolución de las fuerzas que dentro de una ciudad portuaria regional operan en un sentido o en otro, es decir, aquellos actores que son funcionales o adhieren o promueven un sentido de esa fuerza u otra, incidiendo en:

a) conformación de los discursos

b) en la formación de la opinión pública y más puntualmente en las políticas adoptadas en el sistema portuario argentino, tanto en la formación de los recursos humanos y la actuación política. 
¿Y porque especialmente necesario conocer esto y su correlato en la administración pública? Fundamentalmente para entender que ha sucedido con la preservación del patrimonio histórico de los puertos; las principales dificultades en la construcción de políticas de preservación del patrimonio portuario en general.

El caso de Rosario es elocuente. Desde mediados del siglo XIX y hasta 1937 la dinámica del puerto había girado en una misma dirección, insertando cada vez más a la región en el sistema capitalista mundial, hasta que la la convulsión provocada por la Segunda Guerra Mundial, tanto en el comercio internacional como en la comercialización agrícola regional, presentó el desalentador panoráma de los muelles vacíos y una actividad reducida a la mínima expresión.

Rosario perdió su posición de principal puerto exportador de cereales del país en 1939 conociendo así el final de su "época de esplendor". El puerto del librecambio pasó a ser en adelante un puerto dependiente del dirigismo estatal. Ante la gravedad de los acontecimientos internacionales el gobierno argentino consideró ineludible profundizar la centralización de la administración portuaria, la principal fuente de ingreso que disponía, tal como ocurría en otros países. El puerto de Barcelona fue controlado por el franquismo (al igual que él de Rosario recién recuperaría a finales de la década del 60 el máximo movimiento de tráfico registrado en la del 30); y el de Génova se integró a la nueva República Italiana. Asimismo, el puerto de Rosario sobrellevó, además de la disminución de la demanda exportadora mundial, una sumatoria de variables que incidieron negativamente en su condición de ultramar. Solamente tomando en cuenta la competencia de los sistemas de transportes: la conveniencia tarifaria de fletar los productos a través de los ferrocarriles amparados en una legislación parabólica; el avance incontenible y creciente del tráfico de automóviles de carga como competidor del transporte ferroviario y naval; la inauguración de rutas asfaltadas; la vertiginosa expansión de las industrialización nacional por el proceso de sustitución de importaciones, que modificó el escenario económico, social, cultural y político de la década del 40; y fundamentalmente la no intensificación de las tarea de dragado y Canalización de Martín García; y el establecimiento de nuevos puertos privados industriales en la zona del Gran Rosario.

Tales son las implicancias de un estudio integrado entre historia de las relaciones internacionales, el comercio exterior, la economía regional, y las políticas públicas para el desarrollo de las regiones.

Sin embargo, no abundan este tipo de análisis ni los estudios comparados de la historia de las ciudades puertos argentinas, más aún a partir del siglo XIX. Fue por eso que años atrás, cuando Mario Rapoport, autor de una completa y didáctica historia de Buenos Aires, me sugirió que la cotejara con el caso rosarino, así lo hice; más teniendo en cuenta que en ambas ciudades, por su condición de puerto internacional, se reflejaban cabalmente las mencionadas posturas librecambistas y proteccionistas en cuanto al comercio exterior.

Por otra parte, la historia de los puertos es una herramienta de gran versatilidad para comprender la disputa entre las ciudades portuarias por las áreas de influencias que profundizaron las asimetrías en el desarrollo de la Nación y la gran región del Cono Sur. Estudiar por ende a las ciudades puertos regionales implica 
conocer la configuración del espacio y el territorio, y en este sentido hay renovados trabajos sobre el espacio rioplatense y del litoral.

Producida la Revolución de Mayo, en 1810 se profundizará la disputa entre las dos ciudades puertos más importantes del Río de la Plata: Buenos Aires y Montevideo por el control de la cuenca del Plata; en una competencia en pos de hegemonizar el flujo comercial. Aunque en esta relación no podemos ignorar la acción de las elites portuarias del sur del Brasil y de Asunción del Paraguay. La convivencia entre los puertos de Buenos Aires y Montevideo fue conflictiva, a la manera de esos matrimonios por compromiso. Cada uno sacó provecho de sus ventajas.

Desde un enfoque de la historia institucional y política, la historia de la gestión de los puertos de la Argentina a partir de la Revolución de Mayo de 1810 ha sido objeto de distintas periodizaciones. A los fines de esta intervención podemos señalar esquemáticamente las siguientes grandes etapas: La etapa librecambista, que se abre en 1810 y continúa hasta 1943, año en que se nacionaliza el sistema portuario. Etapa que a su vez presenta dos escenarios muy definidos: por un lado, Buenos Aires como puerto único hasta la caída del gobernador de Buenos Aires Juan Manuel de Rosas, en 1852, y por otro la apertura de los ríos interiores a la navegación internacional. En este extenso período de 133 años se permitió la construcción, concesión, y explotación de los puertos por el capital privado, o a provincias y municipios. El Estado asumió obras de infraestructuras tales como las que permitían los accesos a los puertos, muelles y el almacenamiento de la producción. Es la etapa a la que se identifica con la instauración, consolidación y expansión del modelo agroexportador.

La segunda etapa en la gestión de los puertos argentinos, de medio siglo de duración, se habría iniciado en la década del 40 del siglo XX, y concluiría en la década del 90, y es coincidente con la posesión y administración de los puertos exclusivamente desde la Capital Federal. Coincidió en sus inicios con el modelo de sustitución de importaciones, con una orientación marcada hacia el mercado doméstico. Por imperio del comercio internacional a partir de finales de la década del 50 se autorizaron los primeros puertos privados del complejo agroindustrial, coincidente con la apertura del mercado financiero ante la liquidez de la economía internacional pero la inestabilidad económica interna por el nivel de importaciones y la reiteración los déficits fiscales.

La tercera etapa habría tenido lugar a partir de 1991, y se caracterizaría por la descentralización y privatización del sistema portuario, en el contexto del régimen de convertibilidad interno y un modelo neo exportador en el mercado internacional, y recién en ella se vendría a operar un cambio en función a la secular acción monopolizadora del puerto de Buenos Aires.

Un hito en el sistema portuario es la promulgación de la ley 22.080 de política portuaria, y la Ley 22.108, ambas de 1970, abrieron la posibilidad de la existencia de "puertos específicos" operados en forma privada, y elevadores de granos, que operaban en puertos estatales, pudieron actuar prestando servicios de carga en forma comercial por empresas privadas. Es así que empresas privadas comercializadoras de granos pudieron ser propietarias de elevadores terminales. Esto brindó la posibilidad del traspaso de instalaciones portuarias en desuso a cooperativas agrarias y 
asociaciones de productores. Se comenzó a construir numerosas terminales para agrograneles sobre el Río Paraná. Se habilitó el Canal Emilio Mitre que vincula el Río de la Plata con el Paraná de las Palmas y mejora el acceso al Paraná.

Las dos últimas etapas, de creciente privatización del sistema portuario y de un progresivo corrimiento del Estado a un estado pasivo se caracterizan por operar dentro de una tendencia mundial de globalización y modernización.

En 1989, la sancionó la ley N. 23.696 de Reforma del Estado que a través del decreto 20749 estableció los lineamientos para "descentralizar y provincializar" los servicios prestados por la Administración General de Puertos. En 1991 se suspendieron los gravámenes sobre los fletes de exportación e importación, y la liberalización del mercado de fletes cautivos, de pilotaje y cabotaje. Mediante el decreto 817/92 de desregulación marítima y portuaria se disolvió la Capitanía General de Puertos, se declaró en liquidación a la Administración General de Puertos reemplazándola por la Subsecretaría de Puertos y Vías Navegables y se liberalizó el trabajo portuario. Se crearon las administraciones de los puertos de Rosario, Buenos Aires, Quequén, Bahía Blanca, Santa Fe, Ushuaia, como paso necesario para la transferencia de puertos a las provincias hasta que, el 3 de junio de 1992, se dictó la Ley 24.093 de Actividades Portuarias, que contemplaba la privatización de los puertos argentinos, siendo el primera el de Bahía Blanca, siguiéndolo en 1994 el ENAPRO, en Rosario como complejo multipropósito para la transferencia de cargas; y en diciembre del mismo año el Puerto de Buenos Aires, que quedó dividido en tres unidades autónomas: Dock Sud, Puerto Dársena Sur y Puerto Nuevo.

A su vez, y relacionada con la periodización anterior puede resumirse que la evolución de las instalaciones portuarias en Rosario pueden dividirse en cinco etapas bien definidas: La primera, "sin muelles artificiales", desde la época hispana hasta 1856, año en que se constituye el primer proyecto portuario, el de Hopkins (la villa transformada en ciudad había triplicado su población de 3 mil a 9 mil habitantes; la segunda, desde ésta fecha a 1905, en que se habilita el puerto moderno de Rosario (la ciudad ya llegaba a 150 mil habitantes, con todas las complejidades de la ciudad moderna); la tercera, desde 1905 a 1942, en la que el mismo queda bajo la concesión y explotación de una empresa privada (cuando la urbe pasaba los 400 mil habitantes); la cuarta, desde 1942 hasta 1994, en la que permaneció en la órbita del Estado nacional; (la ciudad de 800 mil personas) y la quinta, desde esa última, en que la nación transfirió el puerto a la provincia de Santa Fe y este a su vez confió la administración al Ente Administrador Puerto Rosario (ENAPRO), hasta nuestros días.

La creciente producción cerealera del sur santafesino y en especial del hinterland de la ciudad puerto de Rosario colocó a esta zona en condiciones de generar nuevos emprendimientos, y las terminales portuarias privadas construidas a finales de los sesenta fueron habilitadas en 1978. A partir de entonces empresas multinacionales terminaron de dar forma al complejo portuario de San Lorenzo y Puerto San Martín.

En la segunda mitad del siglo XX, se impusieron nuevos patrones en la operatoria del comercio internacional naval que condujeron a la relocalización de las 
instalaciones portuarias y a la refuncionalización de las que, emplazadas en los centros de las ciudades, quedaron en desuso. La recuperación de estos espacios impulsó visiones retrospectivas de la relación "ciudad puerto", advirtiéndose en distintas disciplinas científicas un creciente interés por "comprender la estructura funcional de las ciudades portuarias" y el desarrollo las regiones. Esta inquietud se tornó cada vez más apremiante cuando hacia la década del 80 y 90, se aceleraron "las innovaciones tecnológicas" y las reglas aperturistas del comercio internacional requirieron acelerar las transformaciones en la morfología de los frentes portuarios.

La utilización del concepto "ciudad puerto" se ha acentuado en las últimas tres décadas por la implementación de programas financiados por organismos internacionales dedicados al mejoramiento de la calidad de vida de las ciudades y por ende al estudio de la secuencia de interfaces entre una ciudad y un puerto, en el espacio urbano, marítimo y fluvial. Se trata de una compleja zona de mediación de funciones locales, regionales e internacionales

Poco después de la expansión de los enfoques promovidos desde la geografía y el urbanismo, comenzaron a difundirse trabajos teóricos de especialistas españoles que propusieron estudiar metodológicamente el devenir de las ciudades portuarias.

Destacado aporte fue el de Agustín Guimerá Ravina y Fernando Monge a fines de los 90 al observar a la ciudad portuaria desde lo sociocultural, la identificación del desarrollo de una estructura social con el surgimiento de nuevas posibilidades y por ende de una dinámica específica de acuerdo a las necesidades del puerto y a las características de los productos comercializados. Monge, quién priorizó una visión antropológica de los estudios portuarios incluyó el análisis los sujetos individuales, descuidados por la historiografía portuaria tradicional. Lo resumió en la siguiente afirmación: "Todo puerto es en sí mismo una comunidad humana".

Las ciudades portuarias encierran una dimensión regional imposible de eludir porque "a manera de cascada", transformaron "el entorno, su gente y la cultura", sostiene Guimerá Ravina. A ellas convergieron elementos que le otorgaron una capacidad generadora de procesos de cambio y modernización. "Haz de fuerza", denomina el autor a esta función que bien podría ser comparada, en mi opinión, a la intensidad de un "haz de luz". En tal sentido el precursor de estos estudios, Broeze, argumenta que cada ciudad puerto tiene un propio ritmo de desarrollo el que podría ser evaluado según el grado de "interrelación de todas las fuerzas" sobre las que ejerce un rol de mediación.

Guimerá Ravina sugiere que mientras el término "ciudad puerto" comprendería el estudio de dos realidades no definitivamente integradas, referirse a "ciudad portuaria" implicaría el análisis de una situación consumada, simbiótica en la que el puerto es la razón de ser de la ciudad pudiendo modificarse y dar paso a una tipología distinta, cuando otras funciones, administrativas, políticas y culturales sobrepasaran en tamaño y alcance a las propiamente portuarias.

La puesta en valor del patrimonio histórico de las Ciudades Puertos Regionales, socializado a escala urbana como una cátedra a cielo abierto a consecuencia del reciclado de las instalaciones portuarias, es clave para el desarrollo sustentable local y global. 
Quizás una de las mayores y primeras intervenciones culturales de gran envergadura realizada a escala mundial que redundó en el diálogo ciudad puerto fue el Complejo Cultural Parque de España, en Rosario, y significó la emergencia de un nuevo paradigma en la intervención de la ciudad. Contemporáneamente al proyecto del Parque España se fue produciendo el reciclaje de Puerto Madero y la transformación del Port Vell de Barcelona, que se convirtió en una referencia internacional para los urbanistas que en las últimas décadas trabajaron por la recuperación del frente marítimo de sus ciudades.

El diálogo ciudad puerto implica una constante búsqueda y redefinición de la identidad urbana, para lo que es necesario contemplar a la misma como un proceso.

La ciudad puerto regional no tiene garantizado un futuro de bienestar por así serlo. El estudio de la evolución histórica de las interfaces puede de alguna manera explicar el presente y advertir su futuro. Se evidencia la conveniencia de una metodología que la contemple bajo el paradigma de la complejidad. Porque la ciudad puerto es como la complejidad misma, una unidad interrelacionada, que requiere necesariamente de enfoques transdisciplinarios (superando la especialización positivista) y la plurisdisciplinariedad para poder abordar al sistema ciudad puerto como un todo organizador, de interacciones, retroacciones, e interretroacciones que se organizan "por sí".

Son en las ciudades portuarias donde es elevadísimo el grado de interconexión y por lo tanto mayores son los requerimientos para abordar el conocimiento de la realidad y tomar las decisiones más lúcidas para la comprensión y resolución de las contingencias del presente.

En función de lo aquí reseñado quiero concluir que desde los primeros trabajos publicados específicamente sobre la historia del puerto de Rosario, comenzando con García Meira en la década del 30, pasando por Juan Jorge Gschwind en los 50, Oscar Ensinck en los 70, y Marta Frutos en los 80 (sin contar con las referencias sobre el puerto que en el siglo XX se desprenden de los trabajos de Juan Álvarez, Miguel Ángel De Marco, Nidia Areces, Marta Bonaudo, Myriam Stanley, Ricardo Falcón, Alicia Megías, Griselda Tarragó, etc. No quiero pronunciar más autores para no incurrir en omisiones) hasta en el primer libro de mi autoría sobre el puerto de Rosario hace quince años atrás, predominan enfoques efectuados desde la especialización o el campo temático del autor.

Con la evolución historiográfica reseñada que se opera mundialmente a partir de los 80 y 90, muy especialmente a través del concepto de ciudad puerto y ciudad portuaria que se ha desarrollado desde fines de los 90 al presente, es que hemos iniciado un camino de integración interdisciplinar para la comprensión de la historia del puerto de Rosario. Quizás como resultado de esa motivación es mi último libro referido a la temática: "Ciudad puerto, universidad y desarrollo regional, Rosario, 1919 y 1968 ". A diferencia de mis anteriores libros "La batalla por el puerto de Rosario" y "El puerto de los rosarinos", centrados en una evolución institucional, se 
incorpora una interpretación sustentada en aportes de distintas disciplinas para explicar ya no sólo la historia del puerto de Rosario sino la relación con distintas interfaces.

Es un camino nuevo, de no más de tres años, y que ha impulsado instancia de cooperación interdisciplinar a través de distintos proyectos que, en el orden provincial, se ha efectuado a través del denominado "Puertos de la Provincia de Santa Fe", y que ha involucrado a autoridades y profesionales de ciudades puertos santafesinas, y del que hemos tenido el placer de contar con el concurso del CEASA y la licenciada Mónica Valentini. Otra actividad de notorio corte interdisciplinar fue la organización de las VI Jornadas Internacionales de Estudios Portuarios, que se realizaron en agosto pasado en Rosario, y en la se presentaron más de 70 propuestas de ponencias, las que se expusieron en las mesas por temas: Economía y Sociedad, Espacio y Territorio, Cultura y Patrimonio, Relaciones Internacionales; Gobernanzas y Políticas Públicas. En el CD con las actas de esas ponencias, se encuentra en ciernes, solo falta agruparla temáticamente, la nueva historia interdisciplinar del puerto de Rosario.

Las conclusiones del encuentro, aceptadas por unanimidad, se centraron en recomendar la promoción de políticas públicas para la preservación del patrimonio histórico de los puertos, lo que incluye la protección y conservación de la documentación (extremadamente dispersa y en muchos casos en una situación precaria); destacar las posibilidades que ofrecen las investigaciones interdisciplinarias de los estudios portuarios y las ciudades portuarias en la renovación de perspectivas historiográficas y en la comprensión del presente, y por ende poner en común los recorridos de investigación para revisar cuestiones de periodización, escalas de análisis, configuración territorial, marcos teóricos, para lograr una articulación, puntos de encuentro entre la historia social, económica y política.

Siguiendo con ese objetivo es que estamos conformando un equipo de las Ciudades Puertos Regionales, con investigadores de Conicet y distintas universidades, y profesionales de distintas áreas. En función de esto se ha conformado además un Foro Internacional de Ciudades Portuaria.

Muchas gracias.

Recibido: 9 de junio de 2016.

Aceptado: 23 de junio de 2016. 